\title{
Erratum to: Third-order dispersion role on parabolic pulse propagation in dispersion-decreasing fiber with normal group-velocity dispersion
}

\author{
S. Zhang • G. Zhao • A. Luo • Z. Zhang
}

Published online: 27 April 2010

(c) Springer-Verlag 2010

Erratum to: Appl Phys B (2009) 94: 227-232

DOI 10.1007/s00340-008-3314-x

Unfortunately, several errors have occurred in our paper published recently.

The correct values are as follows.

- The following values should be used throughout the article:

$$
\begin{aligned}
b= & \frac{\beta_{3} \Gamma_{0}^{2}}{54 \beta_{2}^{3}}, \quad m=-\frac{2 \beta_{3} \Gamma_{0}}{9 \beta_{2}^{2}}, \quad n=-\frac{\beta_{3}^{2} \Gamma_{0}^{2}}{36 \beta_{2}^{4}}, \\
q= & -\frac{\beta_{3}^{4} \Gamma_{0}^{4}}{1944 \beta_{2}^{8}}, \\
\varphi= & \varphi_{0}+\frac{\beta_{3}^{2} \Gamma_{0}^{2} \xi}{54 \beta_{2}^{3}}+\frac{3 \gamma A_{0}^{2}}{2 \Gamma_{0}} \exp \left(\frac{2}{3} \Gamma_{0} \xi\right), \\
\phi= & -\frac{\Gamma_{0}}{6 \beta_{2}} T^{2}+\frac{\beta_{3} \Gamma_{0}^{2}}{54 \beta_{2}^{3}} T^{3}+\varphi_{0}+\frac{\beta_{3}^{2} \Gamma_{0}^{2} \xi}{54 \beta_{2}^{3}} \\
& +\frac{3 \gamma A_{0}^{2}}{2 \Gamma_{0}} \exp \left(\frac{2}{3} \Gamma_{0} \xi\right),
\end{aligned}
$$

The online version of the original article can be found under doi:10.1007/s00340-008-3314-x.

\section{S. Zhang $(\bowtie) \cdot$ G. Zhao $\cdot$ Z. Zhang}

College of Physics Science and Information Engineering, Hebei

Advanced Thin Films Laboratory, Hebei Normal University,

Shijiazhuang 050016, China

e-mail: zhangsm_sd@126.com

Fax: +86-311-86268314

A. Luo

Laboratory of Photonic Information Technology, School of Information and Optoelectronic Science and Engineering, South

China Normal University, Guangzhou 510006, China

$$
\begin{aligned}
& \delta \omega=-\frac{\partial \phi}{\partial T}=\frac{\Gamma_{0}}{3 \beta_{2}} T-\frac{\beta_{3} \Gamma_{0}^{2}}{18 \beta_{2}^{3}} T^{2}, \\
& \beta_{3}=6.05 \times 10^{-3} \mathrm{ps}^{3} / \mathrm{km}, \quad \gamma=3.33(\mathrm{~W} \cdot \mathrm{km})^{-1}, \\
& W_{\text {in }}=32.3 \mathrm{pJ}, \quad \beta_{22}=-0.4485 \mathrm{ps}^{2} / \mathrm{km} .
\end{aligned}
$$

- The following values should be used in Sect. 4.1 and in Fig. 3:

$$
\begin{aligned}
& \beta_{32}=-2.1 \times 10^{-3} \mathrm{ps}^{3} / \mathrm{km}, \quad T_{\mathrm{FWHM}}=97.7 \mathrm{fs}, \\
& P_{\text {peck }}=4283 \mathrm{~W}, \quad T_{\mathrm{FWHM}} / T_{\text {comp }}=40.98 .
\end{aligned}
$$

- The following values should be used in Sect. 4.2:

$T_{\mathrm{FWHM}} / T_{\text {comp }}=39.06, \quad P_{\text {peck }}=4280 \mathrm{~W}$.

- The following value should be used in Sect. 4.3 and in Fig. 5:

$\beta_{32}=-3.5 \times 10^{-3} \mathrm{ps}^{3} / \mathrm{km}$.

The correct version of the figures is as follows. 


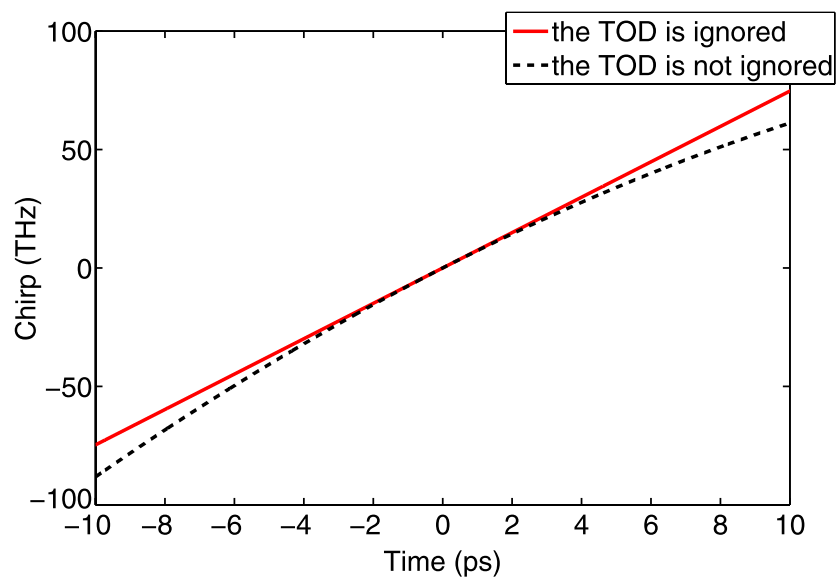

Fig. 1 The frequency chirp for the case when TOD is ignored (solid line $)$ and is not ignored $\left(\beta_{3}=6.05 \times 10^{-3} \mathrm{ps}^{3} / \mathrm{km}\right.$, dotted line $)$
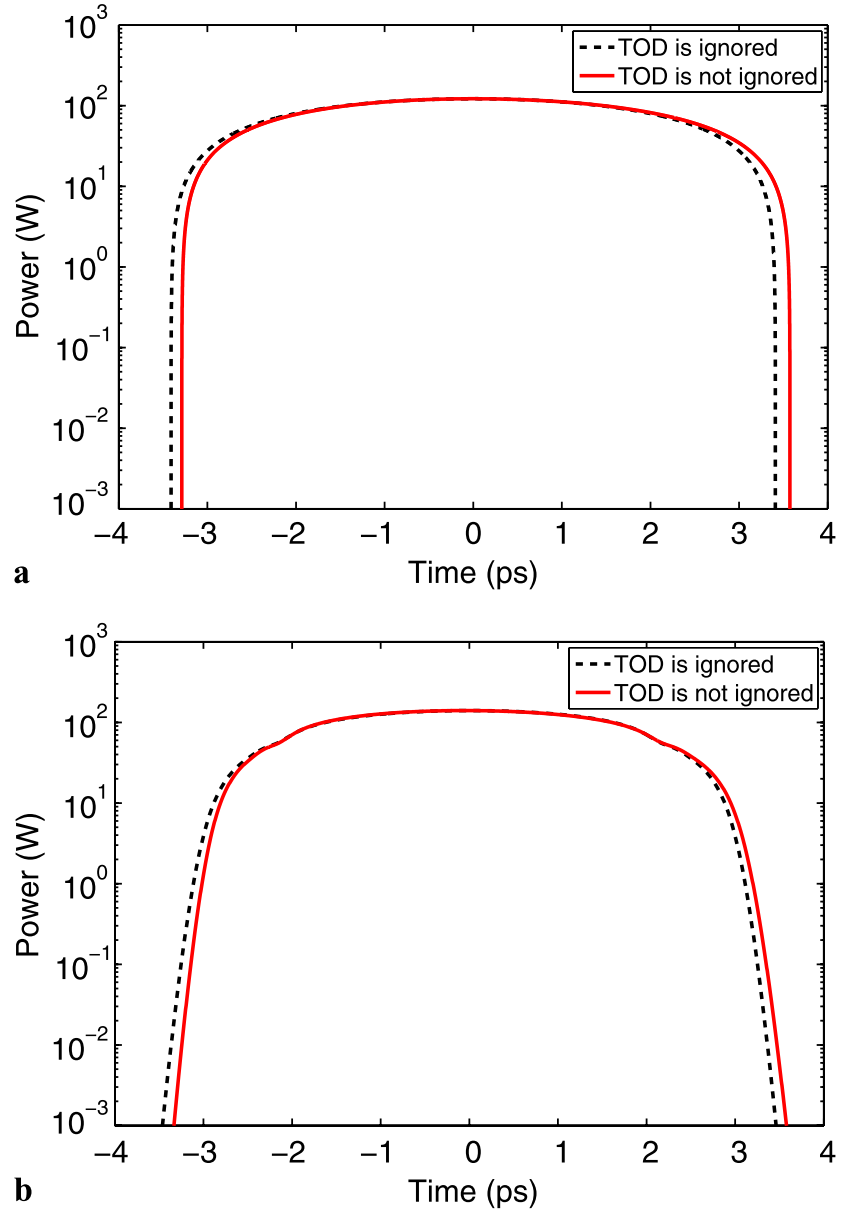

Fig. 2 (a) The theoretically calculated self-similar pulse shapes when TOD is included $\left(\beta_{3}=6.05 \times 10^{-3} \mathrm{ps}^{3} / \mathrm{km}\right.$, solid line $)$ and when the effect of the TOD is not included (dotted line). (b) The corresponding simulation waveforms of the output pulses after propagation over $2 L_{d}=0.576 \mathrm{~km}$

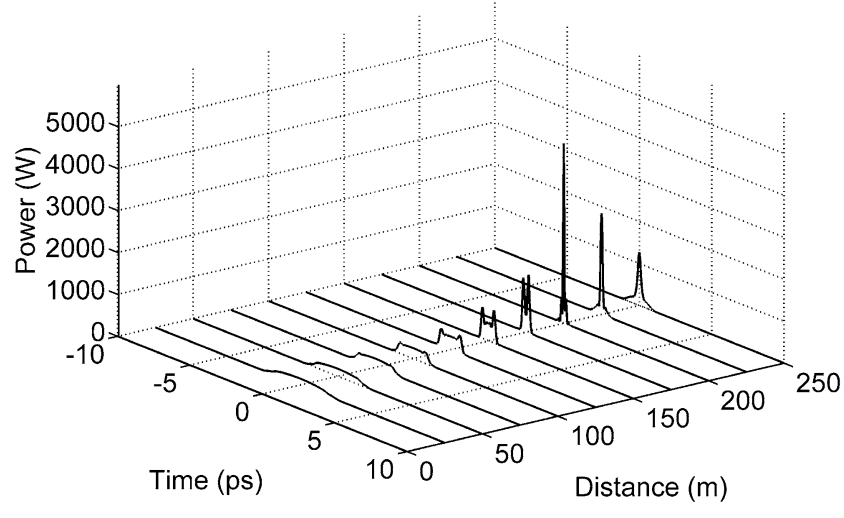

Fig. 3 The evolution of the asymmetric pulse (solid line in Fig. 2(b)) in a dispersion compensation fiber with the full TOD compensation for which the GVD and the TOD are $\beta_{22}=-0.4485 \mathrm{ps}^{2} / \mathrm{km}$ and $\beta_{32}=-2.1 \times 10^{-3} \mathrm{ps}^{3} / \mathrm{km}$, respectively

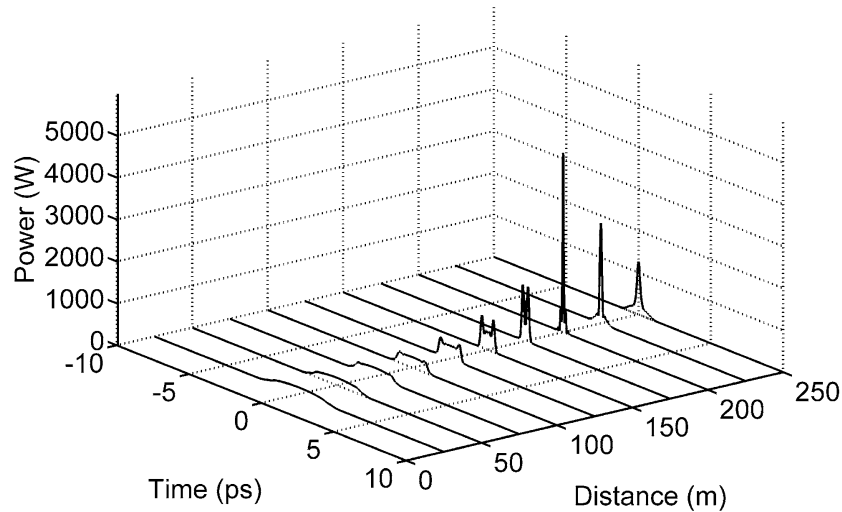

Fig. 4 The evolution of the self-similarity pulse (dotted line in Fig. 2(b)) in a dispersion compensation fiber when TOD is ignored. The GVD and the TOD of the compensation fiber are $\beta_{22}=-0.4485 \mathrm{ps}^{2} / \mathrm{km}$ and $\beta_{32}=0$, respectively

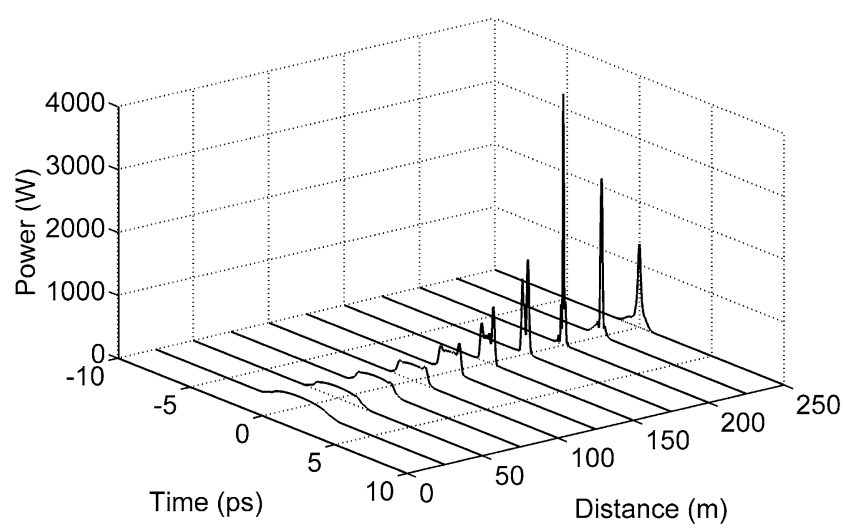

Fig. 5 The evolution of the asymmetric pulse (solid line in Fig. 2(b)) in a dispersion compensation fiber with partially compensated of TOD $\beta_{32}=-3.5 \times 10^{-3} \mathrm{ps}^{3} / \mathrm{km}$ 


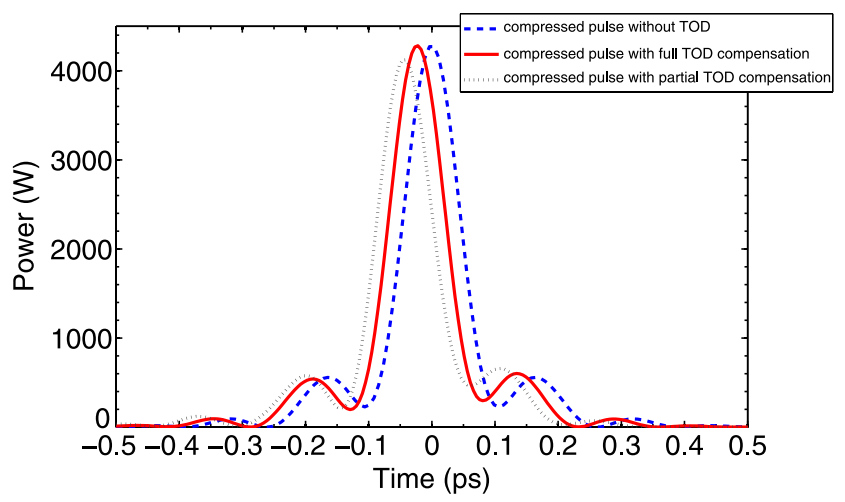

Fig. 6 Waveforms of the compressed pulses when TOD is ignored (dashed line), with the full TOD compensation (solid line), and with the partial TOD compensation (dotted line) 